\title{
How does yeast respond to pressure?
}

P.M.B. Fernandes

\author{
Correspondence \\ P.M.B. Fernandes \\ Departamento de Ciências Fisiológicas \\ UFES \\ Av. Marechal Campos, 1468 \\ 29040-090 Vitória, ES \\ Brasil \\ Fax: +55-27-3335-7342 \\ E-mail:pmbfernandes@gmail.com \\ Presented at the 3rd International \\ Conference on High Pressure \\ Bioscience and Biotechnology, \\ Rio de Janeiro, RJ, Brazil, \\ September 27-30, 2004. \\ Research supported by CNPq. \\ $\ldots \ldots \ldots \ldots \ldots \ldots$
}

Received January 21, 2005

Accepted May 12, 2005
Departamento de Ciências Fisiológicas, Universidade Federal do Espírito Santo, Vitória, ES, Brasil

\begin{abstract}
The brewing and baking yeast Saccharomyces cerevisiae has been used as a model for stress response studies of eukaryotic cells. In this review we focus on the effect of high hydrostatic pressure (HHP) on $S$. cerevisiae. HHP exerts a broad effect on yeast cells characteristic of common stresses, mainly associated with protein alteration and lipid bilayer phase transition. Like most stresses, pressure induces cell cycle arrest. Below $50 \mathrm{MPa}(500 \mathrm{~atm})$ yeast cell morphology is unaffected whereas above $220 \mathrm{MPa}$ wild-type cells are killed. $S$. cerevisiae cells can acquire barotolerance if they are pretreated with a sublethal stress due to temperature, ethanol, hydrogen peroxide, or pressure. Nevertheless, pressure only leads to protection against severe stress if, after pressure pretreatment, the cells are also re-incubated at room pressure. We attribute this effect to the inhibition of the protein synthesis apparatus under HHP. The global genome expression analysis of $S$. cerevisiae cells submitted to HHP revealed a stress response profile. The majority of the up-regulated genes are involved in stress defense and carbohydrate metabolism while most repressed genes belong to the cell cycle progression and protein synthesis categories. However, the signaling pathway involved in the pressure response is still to be elucidated. Nitric oxide, a signaling molecule involved in the regulation of a large number of cellular functions, confers baroprotection. Furthermore, $S$. cerevisiae cells in the early exponential phase submitted to 50-MPa pressure show induction of the expression level of the nitric oxide synthase inducible isoform. As pressure becomes an important biotechnological tool, studies concerning this kind of stress in microorganisms are imperative.
\end{abstract}

Key words

- Saccharomyces cerevisiae

- Stress adaptation

- Gene expression

- High hydrostatic pressure

......................
Saccharomyces cerevisiae is a well-known microorganism that causes bread dough to rise and which is used for the production of alcoholic beverages. Since 1876, when Pasteur demonstrated the essential participation of live yeast in fermentation processes, a huge amount of research has been done on the biochemical reactions related to this process. But yeast really became popular among biologists with the advent of molecular biology. Yeast has proved to be a good eukary- otic model and evidence exists that mechanisms operating in yeast also occur in complex eukaryotes (1). Thus, fundamental yeast research has very wide applications. There is no other eukaryotic organism in which so many molecular details have been elucidated by biochemical and genetic analysis in different areas of cellular and molecular biology. In 1996 the entire yeast genome was sequenced (2), revolutionizing yeast research.

And, how does yeast respond to pres- 
sure? If we take advantage of semiotics to understand pressure and let semiotics assist us to realize that meaning is not contained in the word but is a complex interplay of codes (3), we may answer this question more easily. According to any dictionary, pressure is a force or influence exerted continuously on or against something. Then, pressure is a stress and therefore the correct question may be; how does yeast respond to stress?

Yet our concern is on particular types of stresses, i.e., the stresses associated with volume changes which influence important physiological and biochemical processes, such as protein dissociation and unfolding, lipid bilayer phase transition and ligand binding. With this in mind, we will understand why, although $S$. cerevisiae exists at atmospheric pressure, hydrostatic pressure-inducible genes and proteins also exist in this organism (4-6). Most probably this is a result of overlapping effects of hydrostatic pressure and other stresses for which adaptation mechanisms exist. High hydrostatic pressure (HHP) exerts a broad effect with characteristics similar to those of common stresses, such as temperature, ethanol and oxidative stresses. Hydrostatic pressure represents an interesting form of stress whose study in yeast may shed light on the effect of stresses on other organisms.

Yeast viability during hydrostatic pressure treatment decreases with increasing pressure and this effect is more pronounced when cells are submitted to pressures above 100 $\mathrm{MPa}$, while at $220 \mathrm{MPa}$ all wild-type cells are killed. A pressure of $50 \mathrm{MPa}$ is not sufficient to kill or to alter yeast cell morphology. Yeast cells in the stationary phase, where cells are growth arrested and undergo a variety of morphological and physiological changes, are more resistant to pressure than proliferating cells $(7,8)$. In the budding yeast $S$. cerevisiae the presence of a bud reflects the cell cycle position; cells in the G1 phase of the cell cycle are non-budded and the bud appears at approximately the time when the cell enters the $\mathrm{S}$ phase. When $S$. cerevisiae cells are exposed to a nutrient limitation, mating pheromone or mild stress, HHP included, they arrest in the G1 phase of the cell cycle (9-12). Yeast cells pressurized at $50 \mathrm{MPa}$ for $30 \mathrm{~min}$ and then incubated at atmospheric pressure for 120 min show a decrease in the number of budded cells under the stress condition, reaching a minimum value $45 \mathrm{~min}$ post-pressurization. The cells start recovering after $60 \mathrm{~min}$, but are fully active only after $2 \mathrm{~h}$. Comparing the yeast cell growth response to pressure with the classical stress of heat shock of $40^{\circ} \mathrm{C}$ for $30 \mathrm{~min}$, pressurized cells have a slower response and also take longer to recover normal growth (12). This suggests that even after the yeast cells have been relieved from the pressure stress they still suffer metabolic changes and are responding to this stress.

High hydrostatic pressure affects protein polymerization and also induces protein denaturation, thus interfering with enzyme activity $(13,14)$. Increased protein unfolding and aggregation occur when cells are exposed to high temperature. Several physiological changes take place when yeast cells are exposed to a sublethal heat shock, with a strong induction of heat shock proteins (HSPs) and accumulation of a large cytoplasmic pool of trehalose $(9,15,16)$. In contrast, while high pressure induces the transcription of several stress genes in yeast cells, it does not induce high-molecular weight HSPs or the trehalose 6-phosphate synthase gene (4). Several papers have shown the importance of trehalose content for cell protection against the deleterious effect of HHP (17-20) but a yeast mutant unable to accumulate trehalose acquires barotolerance in the stationary phase or after a heat-shock treatment $(8,17)$. Trehalose is a membrane protector (21) and acts like other membrane protectors that induce tolerance to pressure (22).

Pressure also interferes with cell architecture and cell division, affecting mem- 
brane fluidity and the structure of the cell wall and several intracellular organelles (23). Transmission electron microscopic images of yeast cells suggest that hydrostatic pressure induces changes in the cell wall and cytoskeleton, and thus in the cell membranes and organellar dynamics (8). Indeed, pressure up-regulates HSP12 (4) which codes for a protein related to cell wall flexibility (24), suggesting that pressure directly affects cell wall integrity and the cell responds by production of Hsp12p. Actually, research from the G. Lindsey laboratory has confirmed the cell wall location of Hsp12p, leading to the hypothesis that this highly hydrophilic protein acts on the cell wall as a plasticizer in plastic polymers, interrupting the hydrogen bonding and ionic interactions between adjacent polysaccharide polymers that otherwise result in a stable inflexible structure $(24,25)$.

The effect of pressure on the membranes might be due to the fact that lipids are particularly sensitive to pressure effects, being an order of magnitude more compressible than proteins (26). High pressure orders phospholipid bilayers, causing the fatty acyl chains to pack together more tightly, reducing membrane fluidity (27). This phenomenon also occurs at low temperature (28). An increase in the extent of fatty acid unsaturation can compensate for these effects and maintain the membrane in a functional liquid crystalline state (homeoviscous adaptation). In fact, many deep-sea organisms modulate their membrane fluidity by increasing the proportions of unsaturated fatty acids in response to pressure (29). Nevertheless, unsaturated membranes have a higher degree of disorder than the saturated bilayers, but their motionally disordered regions are barely accessible to water molecules (27), leading to an increase in the baro- or lowtemperature resistance. Stresses that cause membrane destabilization, such as osmotic pressure (30), ethanol (31), cold (32), and hydrostatic pressure $(8,33)$ show a discrete induction of the $E R G 25$ gene $(4,30,32,34)$. Erg25p is a sterol desaturase involved in ergosterol biosynthesis and is suggested to be membrane-bound (35). In the presence of such stresses the cell must maintain membrane integrity by activating mechanisms that are capable of minimizing these deleterious effects. On the other hand, heat-shock down-regulates the ERG25 gene in yeast cells (15). Yet, in yeast it has been shown that the addition of ergosterol induces thermal and ethanol tolerance in sterol auxotrophic strains (36) and vesicles with membranes containing cholesterol have proved to be more resistant to HHP than cholesterol-free ones $(27,37)$. Ergosterol is a sterol containing an unsaturated side chain and cholesterol has a saturated side chain. $S$. cerevisiae cells enriched with ergosterol seem to be more resistant to ethanol than cells enriched with cholesterol (27). Thus, biological membranes have been implicated as a primary sensor of environmental stress and membrane sterols appear to be important in stress tolerance.

The influence of HHP on gene and protein expression has been recognized $(5,38,39)$ and, as DNA microarray technology developed, the expression of yeast genes in response to a variety of physical stimuli has been investigated (15). Moreover, Mentré and Hui Bon Hoa (27), reviewing the effect of HHP on living cells, pointed out that in most cases gene expression is inhibited by pressure but some specific pressure-shock proteins are synthesized and also, regarding DNA stability, HHP shifts from the B form towards the denser $\mathrm{Z}$ form of DNA double helices. Studies from Foguel and Silva's group $(40,41)$ have shown that protein association with DNA is less stable under pressure, demonstrating that high pressure may interfere with transcriptional processes, altering genome expression. This has led some researchers to question whether what has been seen by microarray analysis is not just a matter of differential pressure resistance of 
gene promoter binding or of certain mRNAs. Nevertheless, microarray analysis of yeast cells submitted to HHP has revealed a stress response expression profile. Analysis of the 6,200 known or predicted genes of S. cerevisiae shows that genes involved in stress defense and carbohydrate metabolism are highly induced by pressure, while several genes involved in cellular transcription, protein synthesis and direction and cell cycle regulation are down-regulated by pressure treatment (4). If this global gene expression profile observed under HHP conditions is related to stability under pressure of nucleic acids and proteins that protect cells against the deleterious effects of stresses, would this not be a stress response? On the other hand, if the gene expression profile observed under pressure was just a matter of mRNA resistance, no induction should be seen but only down-regulation, corresponding to the degradation of non-resistant mRNAs. As a matter of fact, considering the published information on yeast response to hydrostatic pressure and other stresses, it is our understanding that the cells have a mechanism to sense the stress, and consequently to activate the gene expression machinery.

Yeast cells are better able to withstand severe stress after they have been exposed to a mild form of stress. Pretreatment with a mild heat stress leads to protection against more severe heat shock and several other stresses, including $\operatorname{HHP}(8,42,43)$. However, a mild heat shock is only one of a number of preconditioning treatments known to induce stress tolerance. Certain chemical agents, osmotic dehydration and nutritional status have effects on yeast thermotolerance (43). In addition, results from our group have shown that hydrogen peroxide, ethanol and cold shock also induce baroresistance (44). Although all of these stresses affect the cell membrane, each of them has its own key target. Protein denaturation is the main cause of death of cells exposed to high temperature (16), while ethanol (45) and hydrogen per- oxide (46) treatment cause oxidative stress, whereas the major problem that yeast cells face at low temperature is the reduction of membrane fluidity. Therefore, the induction of genes related to preservation of the cell membrane, detoxification or protein folding will protect yeast cells against the deleterious effects of high hydrostatic pressure.

$S$. cerevisiae cells submitted to a mild sublethal pressure do not acquire resistance to a subsequent rise to severely high hydrostatic pressure. Baroresistance after barotreatment is only acquired if the cells are incubated at room pressure for a short period of time before the severe HHP stress (17). A short recovery period after pressure treatment is also necessary to enhance survival following a subsequent severe stress shock of high or low temperature. The protective effect is seen after $15 \mathrm{~min}$ of incubation at atmospheric pressure and persists for one hour in the presence of severe pressure and ultra-cold stresses (12). We believe that because most pressure up-regulated genes encode proteins related to membrane protection, and the major problems that result from pressure and cold shock are the reduction of membrane fluidity and impaired protein synthesis, once these membrane protective proteins are synthesized and start playing their role in this defense process, they stabilize the cells for a longer period, resulting in the protection against high pressure and ultralow temperature that is seen even $60 \mathrm{~min}$ after decompression.

In contrast, the protection observed against heat shock is shorter (12). It is well known that the main problem caused by high temperature in living cells is associated with protein folding (16) and pressure does not induce the larger HSPs related to chaperone activity. Pressure, like low-temperature, induces two small HSPs (HSP12 and HSP26) related to membrane destabilization $(4,32)$. The small HSP Hsp26p from $S$. cerevisiae has a molecular chaperone activity and, like other members of the HSP family, protects 
proteins from irreversible aggregation. It has been shown that dissociation of the Hsp26 complex at high temperatures is a prerequisite for efficient chaperone activity (47). The Hsp26p complex might be dissociated under pressure and the initial protection observed could be related to the chaperone activity of this protein. Some time after returning to normal pressure, the complex associates and loses its chaperone activity.

Protein synthesis is indeed one of the most barosensitive cellular functions and it is completely blocked at $67 \mathrm{MPa}$ in $E$. coli and several mammalian cells $(5,27,48)$. Ribosome disassembly is, most probably, the major factor contributing to this inhibition $(49,50)$. On the contrary, RNA synthesis is maintained at pressures at which DNA and protein synthesis are completely inhibited (27). Several groups have reported the specific transcription and translation of genes encoding proteins related to the stress response only after a pressure shock $(5,38)$. In our recently proposed model, genes responsible for stress-inducible proteins that are up-regulated after HHP are unable to synthesize their proteins due to the inhibition of the protein synthesis apparatus during pressurization (12). High pressure causes not only protein synthesis inhibition but also protein denaturation and dissociation, both of which are reversible after pressures up to 100-300 MPa (23). When the cells return to atmospheric pressure after a sublethal pressure treatment the alterations of organelles and of biological processes are rapidly reversed (27) and only then will the newly transcribed mRNAs be translated.

Another feature of HHP is the promotion of vacuole acidification, caused by the dissociation of protons from $\mathrm{H}_{2} \mathrm{CO}_{3}$ or sugar phosphoesters facilitated by the decrease in volume driven by pressure (51). Stresses like hydrostatic pressure, heat shock, ethanol exposure, and osmostress promote cytoplasm acidification in S. cerevisiae (16). Microarray analysis of pressurized yeast cells revealed the induction of $H S P 30$, which codes for a down-regulator of the stressstimulated $\mathrm{H}^{+}$-ATPase $\left(\mathrm{H}^{+}\right.$adenosine triphosphatase) activity. $\mathrm{H}^{+}$-ATPase pumps $\mathrm{H}^{+}$at the expense of ATP depletion. Hsp30p thus plays a role in energy conservation during the stress conditions (52).

The signaling pathways implicated in the pressure response indeed are immediately activated, leading to the induction and repression of a variety of genes, as mentioned above, whose products confer protection to the yeast cell. Nevertheless, exactly which pathways are involved is still under investigation. Gene expression after a diverse range of stressful conditions is commonly regulated by trans-activators that bind to the stress-responsive element that occurs in a large number of gene promoters. However, some genes regulated by Msn2/Msn4p (transactivators), like HSP 12 and $H S P 26$, are expressed under pressure; others, like those implicated in trehalose metabolism, are not. Also, pressure is a particular condition in which HSP3O is up-regulated when HSP 12 and $H S P 26$ also are. HSP3O activation is not related to Msn2/Msn4p or other classical stress transcription factors (52).

Nitric oxide (NO) is a widespread signaling molecule involved in the regulation of a large number of cellular functions (53) and a possible role of $\mathrm{NO}$ as a mediator of the stress response in yeast has been examined $(54,55)$. A cytoprotection is clearly observed when yeast cells are treated with $1 \mathrm{mM}$ of the NO donors sodium nitroprusside and $S$ nitroso- $N$-acetylpenicillamine. Furthermore, $S$. cerevisiae cells in the early exponential phase submitted to 50-MPa pressure showed induction of the expression level of the NO synthase inducible isoform (NOS2). In contrast, regarding NO-induced thermotolerance, heat pretreatment does not lead to an induction of any NOS isoforms (55). It has been observed that NO interacts with and enhances the activity of the transcription factor Ace1p (54), but no other results, to 
our knowledge, have been reported for other transcription factors involved in the NO signaling stress response in yeast.

The global gene expression pattern revealed by the microarray analysis in $S$. cerevisiae submitted to HHP opened up new insights into this unique kind of stress. In addition, the observation that pressure is able to induce protection against pressure and other stresses may be a useful biotechnological tool. Certainly, protection against unfavorable conditions is likely to be controlled by a complex of transcriptional, translational and post-translational mechanisms.
Understanding the connections between different environmental conditions in living cells may contribute to information about the particular signals generated by stresses and may shed light on sensory transduction and biochemical adaptation in eukaryotes.

\section{Acknowledgments}

The author thanks Dr. Mark Culik, INCAPER, for critically reading the manuscript and his students for their help in the laboratory, with special gratitude to Fernando L. Palhano.

\section{References}

1. Haney S, Xu J, Lee Sy et al. (2001). Genetic selection in Saccharomyces of mutant mammalian adenylyl cyclases with elevated basal activities. Molecular and General Genetics, 265: 1120-1128.

2. Goffeau A, Barrell BG, Bussey H et al. (1996). Life with 6000 genes. Science, 274: 546-567.

3. University of Wales Aberystwyth. The MCS site. Chandler D Semiotics for beginners [http://www.abu.ac.uk/media/Documents/S4B/]. Accessed October 26, 2004.

4. Fernandes PMB, Domitrovic T, Kao CM et al. (2004). Genome expression pattern in Saccharomyces cerevisiae cells in response to high hydrostatic pressure. FEBS Letters, 556: 153-160.

5. Gross M \& Jaenicke R (1994). Proteins under pressure. The influence of high hydrostatic pressure on structure, function and assembly of proteins and protein complexes. European Journal of Biochemistry, 221: 617-630.

6. Lammi MJ, Elo MA, Sironen RK et al. (2004). Hydrostatic pressureinduced changes in cellular protein synthesis. Biorheology, 41: 309313.

7. Iwahashi H, Fujii S, Obuchi K et al. (1993). Hydrostatic pressure is like high temperature and oxidative stress in the damage it causes to yeast. FEMS Microbiology Letters, 108: 53-58.

8. Fernandes PMB, Farina M \& Kurtenbach E (2001). Effect of hydrostatic pressure on the morphology and ultrastructure of wild-type and trehalose synthase mutant cells of Saccharomyces cerevisiae. Letters in Applied Microbiology, 32: 42-46.

9. Craig EA (1992). The heat shock response of Saccharomyces cerevisiae. In: Jones EW, Pringle JR \& Broach JR (Editors), The Molecular and Cellular Biology of the Yeast Saccharomyces. Vol. 2. Gene Expression. Cold Spring Harbor Laboratory Press, Cold Spring Harbor, NY, USA.

10. Lew DJ, Weinert T \& Pringle JR (1992). Cell cycle control in Saccharomyces cerevisiae. In: Jones EW, Pringle JR \& Broach JR (Editors), The Molecular and Cellular Biology of the Yeast Saccharomyces. Vol. 1. Gene Expression. Cold Spring Harbor Laboratory Press, Cold Spring Harbor, NY, USA.

11. Wheals AE (1987). Biology of the cell cycle in yeasts. In: Rose AH \& Harrison JS (Editors), The Yeasts. Academic Press Inc., London, UK.
12. Palhano FL, Gomes HL, Orlando MTD et al. (2004). Pressure response in the yeast Saccharomyces cerevisiae: from cellular to molecular approaches. Cellular and Molecular Biology, 50: 447-457.

13. Silva JL \& Weber P (1993). Pressure stability of proteins. Annual Review of Physical Chemistry, 44: 89-113.

14. Silva JL, Foguel D \& Royer CA (2001). Pressure provides new insights into protein folding, dynamics and structure. Trends in Biochemical Sciences, 26: 612-618.

15. Gasch AP, Spellman PT, Kao CM et al. (2000). Genomic expression programs in the response of yeast cells to environmental changes. Molecular Biology of the Cell, 11: 4241-4257.

16. Piper PW (1997). The yeast heat shock response. In: Mager WH \& Hohmann S (Editors), Yeast Stress Response. R.G. Landes Company, Austin, TX, USA.

17. Fernandes PMB, Panek AD \& Kurtenbach E (1997). Effect of hydrostatic pressure on a mutant of Saccharomyces cerevisiae deleted in the trehalose-6-phosphate synthase gene. FEMS Microbiology Letters, 152: 17-21.

18. Fujii S, Iwahashi H, Obuchi K et al. (1996). Characterization of a barotolerant mutant of the yeast Saccharomyces cerevisiae: importance of trehalose content and membrane fluidity. FEMS Microbiology Letters, 141: 97-101.

19. Iwahashi H, Obuchi K, Fujii S et al. (1997). Barotolerance is dependent on both trehalose and heat-shock protein 104 but is essentially different from thermotolerance in Saccharomyces cerevisiae. Letters in Applied Microbiology, 25: 43-47.

20. Iwahashi $\mathrm{H}$, Obuchi K, Fujii S et al. (1997). Effect of temperature and the role of Hsp104 and trehalose in barotolerance of Saccharomyces cerevisiae. FEBS Letters, 416: 1-5.

21. Singer MA \& Lindquist $S$ (1998). Thermotolerance in Saccharomyces cerevisiae: the Yin and Yang of trehalose. Trends in Biotechnology, 16: 460-468.

22. Komatsu Y, Obuchi K, Iwahashi H et al. (1991). Deuterium oxide, dimethylsulfoxide and heat shock confer protection against hydrostatic pressure damage in yeast. Biochemical and Biophysical Research Communications, 174: 1141-1147.

23. Mentré P, Hamraoui L, Hui Bon Hoa G et al. (1999). Pressuresensitivity of endoplasmic reticulum membrane and nucleolus as 
revealed by electron microscopy. Cellular and Molecular Biology, 45: 353-362.

24. Motshwene P, Karreman R, Kgari G et al. (2004). LEA (late embryonic abundant)-like protein Hsp 12 (heat-shock protein 12) is present in the cell wall and enhances the barotolerance of the yeast Saccharomyces cerevisiae. Biochemistry Journal, 377: 769-774.

25. Sales K, Brandt W, Rumbak E et al. (2000). The LEA-like protein HSP12 in Saccharomyces cerevisiae has a plasma membrane location and protects membranes against desiccation and ethanol-induced stress. Biochimica et Biophysica Acta, 1463: 267-278.

26. Weber $G \&$ Drickamer HG (1983). The effect of high pressure upon proteins and other biomolecules. Quarterly Reviews of Biophysics, 16: 89-112.

27. Mentré P \& Hui Bon Hoa G (2001). Effects of high hydrostatic pressures on living cells: A consequence of the properties of macromolecules and macromolecule-associated water. International Review of Cytology, 201: 1-84.

28. Quinn PJ, Joo F \& Vigh L (1989). The role of unsaturated lipids in membrane structure and stability. Progress in Biophysics and Molecular Biology, 53: 71-103.

29. Bartlett DH (2002). Pressure effects on in vivo microbial processes. Biochimica et Biophysica Acta, 1595: 367-381.

30. Yale J \& Bohnert HJ (2001). Transcript expression in Saccharomyces cerevisiae at high salinity. Journal of Biological Chemistry, 276: 15996-16007.

31. Cartwright CP, Veazey FJ \& Rose AH (1987). Effect of ethanol on activity of the plasma-membrane ATPase and accumulation of glycine by Saccharomyces cerevisiae. Journal of Genetic Microbiology, 133: 857-865.

32. Sahara T, Goda T \& Ohgiya S (2002). Comprehensive expression analysis of time-dependent genetic responses in yeast cells to low temperature. Journal of Biological Chemistry, 277: 50015-50021.

33. Perrier-Cornet JM, Hayert M \& Gervais P (1999). Yeast cell mortality related to a high-pressure shift: occurrence of cell membrane permeabilization. Journal of Applied Microbiology, 87: 1-7.

34. Alexandre H, Ansanay-Galeote V, Dequin S et al. (2001). Global gene expression during short-term ethanol stress in Saccharomyces cerevisiae. FEBS Letters, 498: 98-103.

35. Bard M, Bruner DA, Pierson CA et al. (1996). Cloning and characterization of ERG25, the Saccharomyces cerevisiae gene encoding C4 sterol methyl oxidase. Proceedings of the National Academy of Sciences, USA, 93: 186-190.

36. Swan TM \& Watson K (1998). Stress tolerance in a yeast sterol auxotroph: role of ergosterol, heat shock proteins and trehalose. FEMS Microbiology Letters, 169: 191-197.

37. Beney L, Perrier-Cornet JM, Hayert M et al. (1997). Shape modification of phospholipid vesicles induced by high pressure: influence of bilayer compressibility. Biophysical Journal, 72: 1258-1263.

38. Bartlett DH, Kato C \& Horikoshi K (1995). High pressure influences on gene and protein expression. Research in Microbiology, 146: 697-706.

39. Takahashi K, Kubo T, Kobayashi K et al. (1997). Hydrostatic pressure influences mRNA expression of transforming growth factorbeta 1 and heat shock protein 70 in chondrocyte-like cell line. Journal of Orthopaedic Research, 15: 150-158.
40. Foguel D \& Silva JL (1994). Cold denaturation of a repressoroperator complex: the role of entropy in protein-DNA recognition. Proceedings of the National Academy of Sciences, USA, 91: 82448247.

41. Mohana-Borges R, Pacheco AB, Sousa FJ et al. (2000). LexA repressor forms stable dimers in solution. The role of specific DNA in tightening protein-protein interactions. Journal of Biological Chemistry, 275: 4708-4712.

42. Iwahashi H, Kaul SC, Obuchi K et al. (1991). Induction of barotolerance by heat shock treatment in yeast. FEMS Microbiology Letters, 80: 325-328.

43. Piper PW (1993). Molecular events associated with acquisition of heat tolerance by the yeast Saccharomyces cerevisiae. FEMS Microbiology Reviews, 11: 339-355.

44. Palhano FL, Orlando MTD \& Fernandes PMB (2004). Induction of baroresistance by hydrogen peroxide, ethanol and cold-shock in Saccharomyces cerevisiae. FEMS Microbiology Letters, 233: 139145.

45. Costa V, Reis E, Quintanilha A et al. (1993). Acquisition of ethanol tolerance in Saccharomyces cerevisiae: The key role of the mitochondrial superoxide dismutase. Archives of Biochemistry and Biophysics, 300: 608-614.

46. Costa V \& Moradas-Ferreira P (2001). Oxidative stress and signal transduction in Saccharomyces cerevisiae: insights into ageing, apoptosis and diseases. Molecular Aspects of Medicine, 22: 217246.

47. Haslbeck M, Walke S, Stromer T et al. (1999). Hsp26: a temperature-regulated chaperone. EMBO Journal, 18: 6744-6751.

48. Landau JV (1967). Induction, transcription and translation in Escherichia coli: a hydrostatic pressure study. Biochimica et Biophysica Acta, 149: 506-512.

49. Gross M \& Jaenicke R (1990). Pressure-induced dissociation of tight couple ribosomes. FEBS Letters, 267: 239-241.

50. Gross M, Lehle K, Jaenicke R et al. (1993). Pressure-induced dissociation of ribosomes and elongation cycle intermediates. Stabilizing conditions and identification of the most sensitive functional state. European Journal of Biochemistry, 218: 463-468.

51. Abe F, Kato $C$ \& Horikoshi K (1999). Pressure-regulated metabolism in microorganisms. Trends in Microbiology, 7: 447-453.

52. Seymour IJ \& Piper PW (1999). Stress induction of HSP30, the plasma membrane heat shock protein gene of Saccharomyces cerevisiae, appears not to use known stress-regulated transcription factors. Microbiology, 145: 231-239.

53. Monkada S, Palmer RMJ \& Higgs EA (1991). Nitric oxide: physiology, pathophysiology and pharmacology. Pharmacological Reviews, 43: 109-141.

54. Chiang KT, Shinyashiki M, Switzer CH et al. (2000). Effects of nitric oxide on the copper-responsive transcription factor Ace1 in Saccharomyces cerevisiae: cytotoxic and cytoprotective actions of nitric oxide. Archives of Biochemistry and Biophysics, 377: 296-303.

55. Domitrovic T, Palhano FL, Barja-Fidalgo $C$ et al. (2003). Role of nitric oxide in the response of Saccharomyces cerevisiae cells to heat shock and high hydrostatic pressure. FEMS Yeast Research, 3: 341-346. 